\title{
Insights in the ligand shell, the coordination mode and reactivity of carboxylic acid capped metal oxide nanocrystals.
}

\author{
Jonathan De Roo, ${ }^{1 * \#} \underline{\text { Edwin A. Baquero }},{ }^{2 \#}$ Yannick Coppel, ${ }^{3}$ Katrien De Keukeleere, ${ }^{1}$ \\ Isabel Van Driessche, ${ }^{1}$ Céline Nayral, ${ }^{2}$ Zeger Hens, ${ }^{1}$ Fabien Delpech ${ }^{2 *}$ \\ \# Both authors contributed equally. \\ ${ }^{1}$ Department of Inorganic and Physical Chemistry, Ghent University, Gent, Belgium \\ 2 INSA, UPS, CNRS, Laboratoire de Physique et Chimie des Nano-Objets (LPCNO), \\ Université de Toulouse, Toulouse, France. \\ ${ }^{3}$ Laboratoire de Chimie de Coordination, CNRS, UPR 8241, Université de Toulouse, \\ Toulouse, France.
}

*Corresponding authors:

Dr. Jonathan De Roo
Department of Inorganic and Physical Chemistry
Ghent University
Krijgslaan 281-S3
B-9000 Gent
Belgium
Tel: +32-9-2644449
Fax: +32-9-2644983
Jonathan.DeRoo@ugent.be

Prof. dr. Fabien Delpech Laboratoire de Physique et Chimie des Nano-Objets Institut National des Sciences Appliquées 135 avenue de Rangueil 31077 Toulouse cedex 4 tel. $+33(0) 561559650$ Fax. +33 (0) 561559697 FDelpech@insa-toulouse.fr

Jonathan.DeRoo@ugent.be

\begin{abstract}
A detailed knowledge of surface chemistry is necessary to bridge the gap between nanocrystal synthesis and applications. Although it was proposed that carboxylic acids bind to metal oxides in a dissociative $\mathrm{NC}(\mathrm{X})_{2}$ binding motif, this surface chemistry was inferred from indirect evidence on $\mathrm{HfO}_{2}$ nanocrystals (NCs). Here, we show a more detailed picture of the coordination mode of carboxylate ligands on $\mathrm{HfO}_{2}$ and $\mathrm{ZrO}_{2}$ NC surfaces, by direct observation via solid state NMR techniques. Surface adsorbed protons are clearly distinguished and two coordination modes of the carboxylic acid are noted; chelating and bridging. We also find that secondary ligands penetrate the ligand shell and have the same orientation with respect to the surface as the primary ligands, indicating that the ionic or hydrogen bond interactions with the surface are more important than the van der Waals interactions with neighboring ligands. During ligand exchange
\end{abstract}


with amines, the chelating carboxylate is preferentially removed. Finally, we show that the $\mathrm{HfO}_{2}$ and $\mathrm{ZrO}_{2} \mathrm{NCs}$ catalyze the imine formation from acetone and oleylamine. Together with the already reported catalytic activity of hafnium oxide, these results put colloidal metal oxide nanocrystals squarely in the focus of catalysis research.

Keywords: carboxylates, nanocatalysis, nanoparticles, NMR, surface chemistry

\section{Introduction}

Colloidal nanocrystals (NCs) have been synthesized in various sizes, compositions and shapes, ${ }^{[1]}$ all sharing the same fundamental characteristic: a large surface area. The surface can be either manipulated, used or passivated but cannot be neglected for most applications such as catalysis, ${ }^{[2]}$ thermoelectrics, ${ }^{[3]}$ nanocomposites, ${ }^{[4]}$ thin film electronics, ${ }^{[5]}$ optoelectronics ${ }^{[6]}$ or electrochromic devices. ${ }^{[7]}$ The NC surface determines the colloidal stability and the physico-chemical properties of the NCs. For these reasons, the surface is often capped with organic surfactants, hence the identification of a colloidal NC as a hybrid object with an organic-inorganic interface. Characterizing the NC core-ligand interface (i.e., the binding modes, the dynamics and the reactivity of the ligands) demands analytical tools probing the NC surface at the molecular scale. Among the various surface characterization methods such as infrared (IR) and X-ray photoelectron spectroscopy (XPS), nuclear magnetic resonance (NMR) stands out due to its high resolving power. ${ }^{[8]}$

Combining solution ${ }^{1} \mathrm{H}$ NMR and elemental analysis (inductively coupled plasma - mass spectroscopy, ICP-MS), it was established that binary metal chalcogenide nanocrystals such as PbSe and CdSe NCs are cation rich and the charge is balanced by X-type carboxylates in a binding motif denoted as $\mathrm{NC}\left(\mathrm{MX}_{2}\right)$, see Scheme $1 .{ }^{[9]}$ Furthermore, this $\mathrm{MX}_{2}$ unit as a whole, is a Z-type ligand and can also be involved in ligand displacement, having a great impact on the optical properties of the nanocrystals. ${ }^{[6 \mathrm{~d}]}$ In contrast, carboxylic acids are able to dissociate on stoichiometric metal oxide NCs. ${ }^{[10]}$ Presumably, protons bind to surface oxygen atoms whereas carboxylates bind to surface metal sites, a binding motif denoted as $\mathrm{NC}(\mathrm{X})_{2}$, see Scheme 1. This combination of X-type ligands is charge neutral as a whole, and allows for ligand displacement by L-type amine ligands. ${ }^{[11]}$ Furthermore, the surface protons were shown to greatly affect the redox chemistry of $\mathrm{ZnO}$ nanocrystals ${ }^{[12]}$ and determined the catalytic 
activity of $\mathrm{HfO}_{2}$ NCs. ${ }^{[2 \mathrm{a}]}$ Another example of the $\mathrm{NC}(\mathrm{X})_{2}$ motif is found in case of stoichiometric $\mathrm{CsPbBr}_{3}$ perovskite NCs, which are dynamically stabilized by an ion pair: alkylammonium carboxylate (Scheme 1). ${ }^{[13]}$ The deep understanding of NC surface chemistry has led to a rationalization of ligand exchange and has been advancing NC applications. ${ }^{[14]}$ Further elaboration on the LXZ classification, binding motifs and ligand exchange can be found elsewhere. ${ }^{[2 \mathrm{a}, 14]}$

\begin{tabular}{lll}
\hline Example & Binding motif & Schematic presentation \\
\hline $\mathrm{CdSe}\left[\mathrm{Cd}(\mathrm{OOCR})_{2}\right]$ & $\mathrm{NC}\left(\mathrm{MX}_{2}\right)$ \\
$\mathrm{HfO}_{2}(\mathrm{H})(\mathrm{OOCR})$ & $\mathrm{NC}(\mathrm{X})_{2}$ \\
$\mathrm{CsPbBr}_{3}\left(\mathrm{RNH}_{3}\right)(\mathrm{OOCR})$ & $\mathrm{NC}(\mathrm{X})_{2}$
\end{tabular}

Scheme 1. The surface chemistry of NCs, stabilized by carboxylates/carboxylic acids.

Unfortunately, the surface chemistry was often inferred indirectly since proton or carbon nuclei close to or on the surface are not detectable in solution ${ }^{1} \mathrm{H}$ NMR. ${ }^{[15]}$ Although derivatization and ligand exchange are commonly used to characterize ligands, ${ }^{[10,16]}$ they are invasive, ligand-specific processes that may have uncontrolled effects such as surface oxidation or residual water adsorption. ${ }^{[16 c]}$ Furthermore, solution ${ }^{1} \mathrm{H}$ NMR does not allow to determine relations between different ligands and different surface sites (as is the case in the $\mathrm{NC}(\mathrm{X})_{2}$ motif). On the other hand, solid state NMR (ssNMR), especially using the crosspolarization $(\mathrm{CP})$ sequence, is of high relevance in the context of surface characterization and mobility-restricted fragments or ligands: the low motion can be advantageously used through the CP experiment to enhance the signals which are typically too broad and too weak to be observed. This is usually achieved using traditional $1 \mathrm{D}{ }^{1} \mathrm{H}-\mathrm{X} \mathrm{CP}\left(\mathrm{X}={ }^{13} \mathrm{C},{ }^{31} \mathrm{P},{ }^{77} \mathrm{Se} \ldots\right)$ or $2 \mathrm{D}$ ${ }^{1} \mathrm{H}-\mathrm{X}$ heteronuclear correlation (HETCOR).

In this paper, we study two technologically relevant metal oxide NCs; hafnium and zirconium oxide for their use in photoluminescence, ${ }^{[17]}$ catalysis, ${ }^{[2 \mathrm{a}]}$ volume holography, ${ }^{[4 \mathrm{~d}]}$ biomedical applications, ${ }^{[18]}$ superconducting nanocomposites, ${ }^{[4 b]} \gamma$-ray scintillators ${ }^{[4 c]}$ and high $\kappa$ or high refractive index nanocomposites. ${ }^{[19]}$ We use solid state NMR to shed light on the nature of interaction between the NC surface and both primary and secondary ligands. A first group of ligands is covalently interacting with the surface and a second group of ligands is hydrogen 
bonding or electrostatically interacting. In addition, we confirmed the $\mathrm{NC}(\mathrm{X})_{2}$ binding motif that was inferred from solution NMR measurements. ${ }^{[10-11]}$ It is shown that both on $\mathrm{ZrO}_{2}$ and $\mathrm{HfO}_{2} \mathrm{NCs}$, carboxylates have two different coordination modes (chelating and bridging) whereas the bridging mode is clearly favored. We show unambiguously the presence of surface protons and their correlation with the bridging carboxylate mode, uncovering an unprecedented level of detail compared to previous ssNMR studies of carboxylic acid capped $\mathrm{ZrO}_{2}$ and $\mathrm{HfO}_{2}$ particles. ${ }^{[20]}$ After ligand exchange with L-type amine ligands and purification with acetone, the chelating mode is removed and in the supernatant, an imine product is found, indicating a catalytic effect whereas the extent of imine formation is higher for $\mathrm{HfO}_{2}$ NCs.

\section{Results and Discussion}

Nanocrystal synthesis and solution NMR. Aggregated, $\mathrm{HCl}$ capped $\mathrm{ZrO}_{2}$ and $\mathrm{HfO}_{2} \mathrm{NCs}$ were synthesized in benzyl alcohol from the respective metal chloride precursor. ${ }^{[1]}$ As expected, the monoclinic crystal phase is recognized in the XRD diffractogram of both $\mathrm{ZrO}_{2}$ and $\mathrm{HfO}_{2}$ (Figure S1). The NCs were de-aggregated by a post-synthetic surface modification $^{[4 \mathrm{~d}, 10,21 \mathrm{~b}, 22]}$ with dodecanoic acid and oleylamine (Scheme 2). It was earlier inferred from solution ${ }^{1} \mathrm{H}$ NMR experiments ${ }^{[10,21 \mathrm{~b}]}$ that dodecanoic acid is the primary ligand, tightly bound to the NCs in a monolayer. Oleylammonium chloride and excess dodecanoic acid are secondary ligands, interacting weakly with the surface, a state which was vaguely described as "entangled" in the ligand shell or physisorbed. ${ }^{[10,23]}$ These secondary ligands could be removed by extensive purification (Scheme 2).
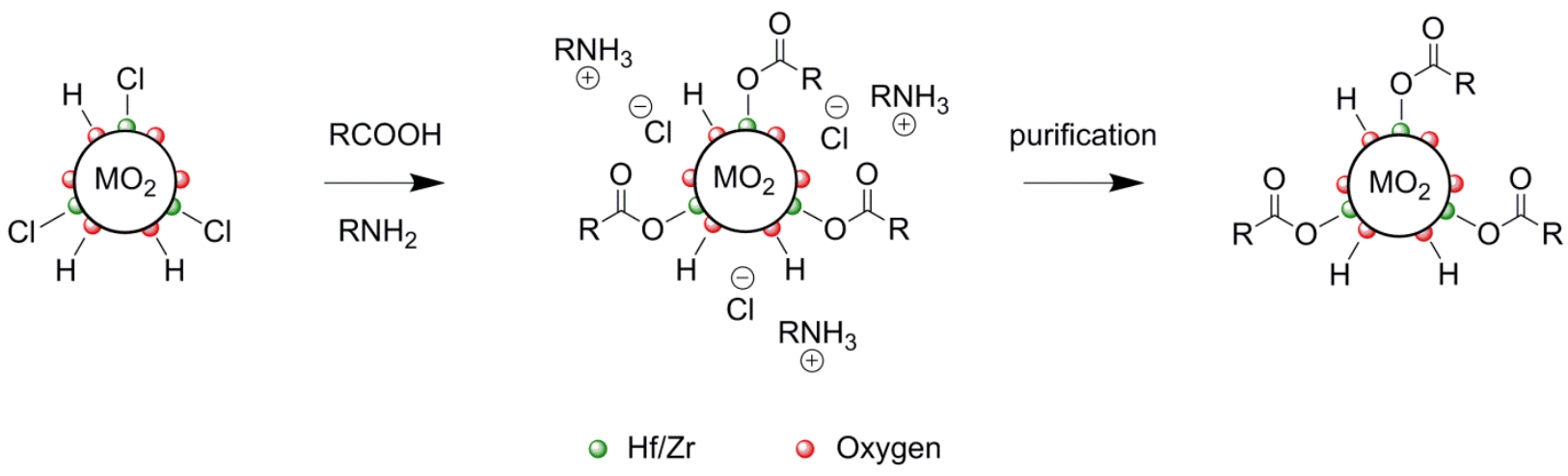

Scheme 2. Surface modification and purification of $\mathrm{ZrO}_{2}$ and $\mathrm{HfO}_{2} \mathrm{NCs}$.

The $\mathrm{HfO}_{2} \mathrm{NCs}$ are about $5 \mathrm{~nm}$ and slightly ellipsoidal while the $\mathrm{ZrO}_{2}$ NCs are more irregularly shaped but also around $5 \mathrm{~nm}$ in size (Figure 1A and 1B). Thoroughly purified NCs feature only broadened resonances in the ${ }^{1} \mathrm{H}$ NMR spectrum (Figure 1C), pertaining to a monolayer of tightly bound dodecanoic acid. The resonances of oleylammonium chloride are 
absent. It is clear that no additional information about the coordination of dodecanoic acid to the surface can be extracted from the spectrum since resonances $\mathbf{1}$ and $\mathbf{2}$ are simply not observed. This finding is not unexpected since tightly bound surfactants have a significantly reduced mobility - inducing line broadening in NMR - and this effect is more predominant the closer protons are located to the surface. Therefore, the first $\mathrm{CH}_{2}$ units next to a functional group (e.g., carboxylic acid) are broadened beyond detection and small ligands are even completely invisible in solution ${ }^{1} \mathrm{H}$ NMR. ${ }^{[15]}$
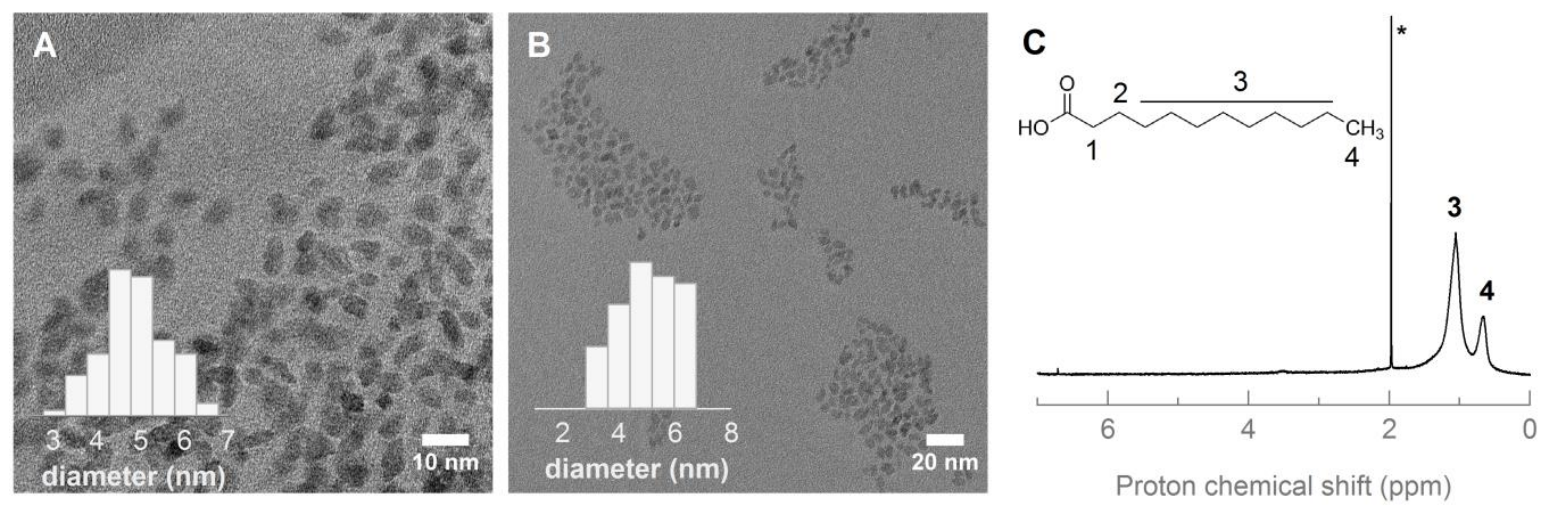

Figure 1. (A) TEM pictures and derived histograms of $\mathrm{HfO}_{2} \mathrm{NCs}$ and (B) $\mathrm{ZrO}_{2} \mathrm{NCs}$. (C) Solution ${ }^{1} \mathrm{H}$ NMR spectrum of a NC dispersion stabilized by dodecanoic acid and purified 5 times with acetone. The resonance denoted by $*$ is attributed to acetone.

$\mathrm{ZrO}_{2}$ - ligand shell configuration. To obtain a more complete picture on the ligand shell, we turned to solid state NMR. Two $\mathrm{ZrO}_{2} \mathrm{NC}$ samples were prepared, either purified five times (5x) by precipitation with acetone or only once purified $(1 \mathrm{x})$. In solid state ${ }^{13} \mathrm{C}$ direct polarization (DP) MAS, all the carbon resonances are probed. Indeed, the carbonyl (181.6 ppm) and $\alpha \mathrm{CH}_{2}$ (37.2 ppm) resonances of dodecanoic acid are observed in the DP MAS spectrum of $5 \mathrm{x}$ purified $\mathrm{ZrO}_{2} \mathrm{NCs}$ (Figure 2). The alkene resonance is not observed, confirming the absence of oleylammonium chloride in the ligand shell. To increase the signal to noise ratio, and to assess the ligand orientation on the surface, cross polarization $(\mathrm{CP})$ is used. As the gyromagnetic ratio of ${ }^{1} \mathrm{H}$ is four times higher than of ${ }^{13} \mathrm{C}$, a magnetization transfer of protons to carbons results in ${ }^{13} \mathrm{C}$ signal enhancement. Interestingly, the enhancement is more efficient in case of restricted mobility, rendering $\mathrm{CP}$ ideal to study ligands attached to NC surfaces. ${ }^{[24]}$ The transfer of polarization takes place at the millisecond time scale so for nuclei with mobility faster than milliseconds, the transfer of polarization (and thus signal enhancement) will not be effective. ${ }^{[25]}$ Therefore, the ${ }^{13} \mathrm{C}$ signal intensities obtained with different polarization transfer schemes, CP, DP and INEPT (Insensitive Nuclei Enhanced by Polarization Transfer) yield information on molecular dynamics. DP yields 
quantitative spectra of all ${ }^{13} \mathrm{C}$ nuclei in the sample, while CP and INEPT select molecular segments being rigid (with order parameter $S>0.5$ and/or rotational correlation time $\tau_{\mathrm{c}}>$ $10 \mu \mathrm{s}$ ) or mobile ( $\mathrm{S}<0.05$ and $\tau_{\mathrm{c}}<0.01 \mu \mathrm{s}$ ), respectively. Comparing the CP and DP MAS spectrum of 5x purified $\mathrm{ZrO}_{2} \mathrm{NCs}$ (Figure 2), we observe a decrease in intensity of the $\mathrm{CH}_{3}$ resonance (13.5 ppm), relative to the $\alpha \mathrm{CH}_{2}$ resonance. On the other hand, in the INEPT MAS spectrum, the $\alpha \mathrm{CH}_{2}$ resonance is absent and the $\mathrm{CH}_{3}$ is the most intense resonance. Since the INEPT experiment probes the part of the sample with mobility, the CP and INEPT experiment both indicate a high mobility for the $\mathrm{CH}_{3}$ and a low mobility for the $\alpha \mathrm{CH}_{2}$. Therefore, the carboxylate functional group is interacting with the surface and the methyl group is much further away from the surface.
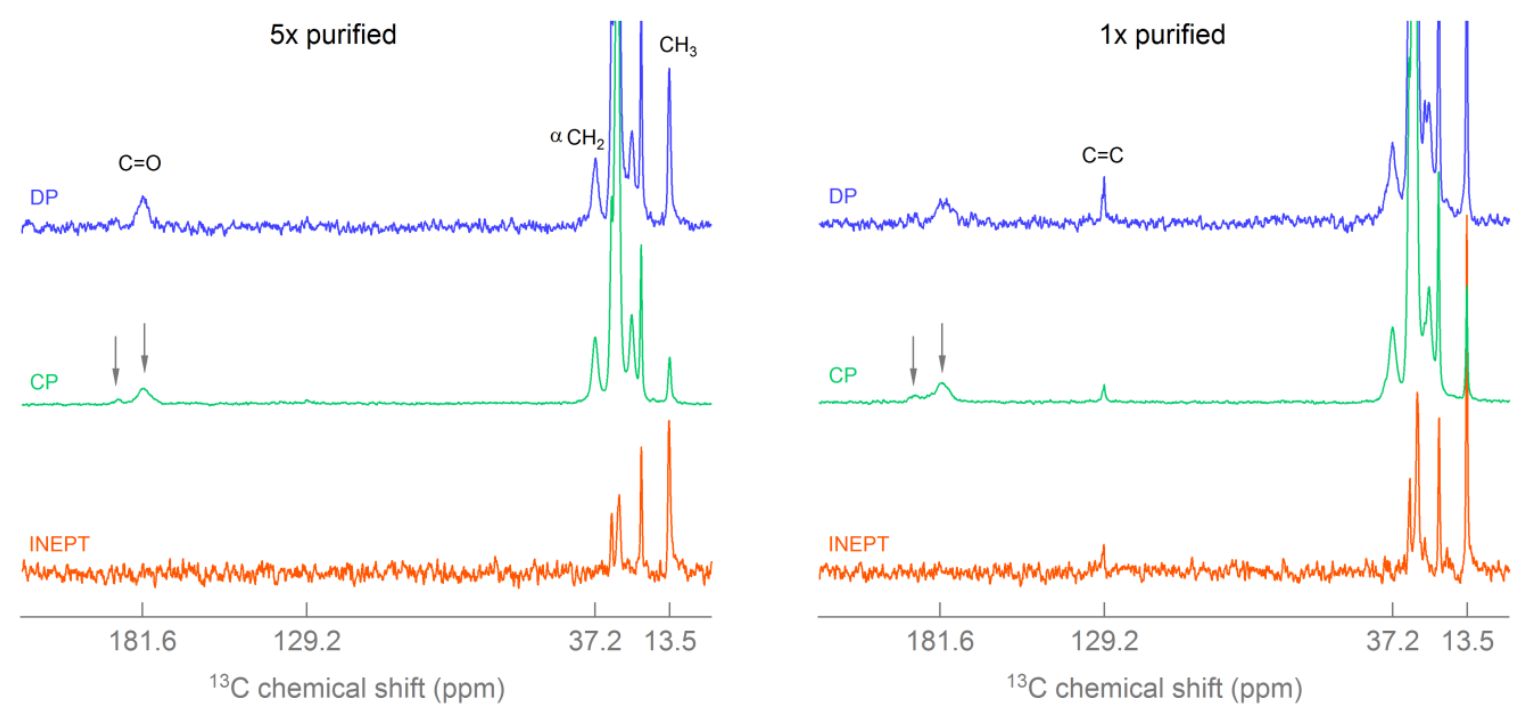

Figure 2. INEPT MAS, ${ }^{1} \mathrm{H}-{ }^{13} \mathrm{C} C P$ MAS and ${ }^{13} \mathrm{C}$ DP MAS spectrum of dodecanoic acid capped $\mathrm{ZrO}_{2} \mathrm{NCs}_{\text {purified once or }}$ five times.

In the spectra of the 1x purified $\mathrm{ZrO}_{2} \mathrm{NCs}$ (Figure 2), there is an additional resonance at 129.2 ppm pertaining to the carbon-carbon double bond of the oleylamino fragment. This indicates that purifying once is not enough to remove the oleylammonium chloride. Most interestingly the $\mathrm{\alpha CH}_{2}$ resonances of both oleylammonium chloride and dodecanoic acid (tightly bound and excess) are not detected in the INEPT experiment, indicating that all $\alpha_{\mathrm{CH}}$ resonances are close to the surface. This proves that the orientation of the secondary surfactants is the same as the orientation of the tightly bound dodecanoic acid; the hydrophilic head towards the surface. This sheds light on the vague term 'entanglement' which was used to described the weakly bound state of surfactants. ${ }^{[10]}$ Since random van der Waals interactions would lead to a random orientation in the ligand shell, we conclude that the second coordination layer is 
interacting through hydrogen bonds or electrostatic interaction with the polar surface or the polar functional group of the tightly bound surfactants.

$\mathrm{ZrO}_{2}$ - ligand binding modes. In the ${ }^{1} \mathrm{H}-{ }^{13} \mathrm{C}$ CP MAS spectra of both $1 \mathrm{x}$ and $5 \mathrm{x}$ purified $\mathrm{ZrO}_{2} \mathrm{NCs}$, the carbonyl region features two signals (at 189.3 and $181.6 \mathrm{ppm}$ ), probably related to two different coordination modes. It is surprising that both modes survived the washing procedure, indicating a strong interaction with the NC surface. Thanks to the frequency difference between the antisymmetric and symmetric $v\left(\mathrm{CO}_{2}{ }^{-}\right)$stretches $\left(130 \mathrm{~cm}^{-1}\right.$, see Figure S2), ionic and monodentate bonding modes can be ruled out since this would require a frequency difference of at least $160 \mathrm{~cm}^{-1} \cdot{ }^{[26]}$ However, it is sometimes misleading to discriminate the two other potential bonding modes (chelating bidentate and bridging bidentate) only via IR data. ${ }^{[27]}$ Fortunately, the carbonyl ${ }^{13} \mathrm{C}$ NMR chemical shift of carboxylate ligands has been shown to be a highly sensitive probe to diagnose with high resolution the binding modes of the carboxylate. ${ }^{[27]}$ The well-documented chemistry of zirconium carboxylates features polynuclear structures, in which the carboxylate ligands can display either a chelating coordination mode or a bridging bidentate one. Both ligation modes are straightforwardly identified in view of the significant difference of the chemical shifts, the chelating mode being shifted 5 to 13 ppm downfield. ${ }^{[28]}$ This shift is in line with previous work on zinc carboxylate where this was explained by electronic effects of the bound metal. ${ }^{[27]}$ It is thus reasonable to assign the resonances at 189.3 and $181.6 \mathrm{ppm}$ respectively to chelating and bridging coordination modes. 

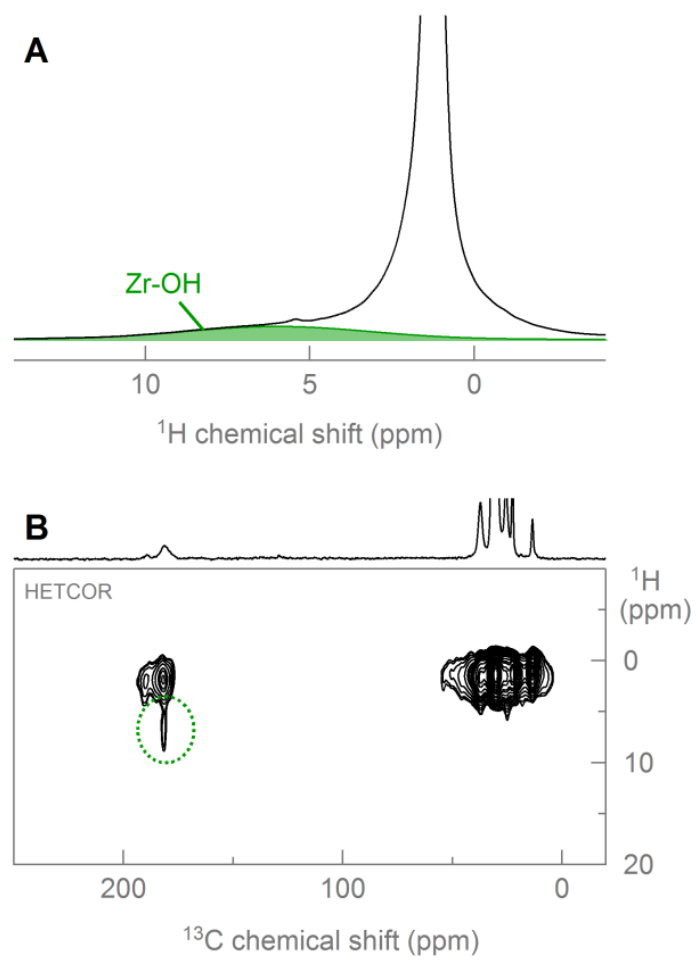

Figure 3. (A) ${ }^{1} \mathrm{H}$ MAS spectrum and (B) ${ }^{13} \mathrm{C}-{ }^{1} \mathrm{H}$ HETCOR of $\mathrm{ZrO}_{2} \mathrm{NCs}$ purified five times.

In the ${ }^{1} \mathrm{H}$ MAS spectrum of the $5 \mathrm{x}$ purified NCs (Figure 3A), a broad contribution was identified next to the expected resonances of dodecanoic acid. We attribute this broad resonance (in green) to protons on the $\mathrm{ZrO}_{2} \mathrm{NC}$ surface, denoted as $\mathrm{Zr}-\mathrm{OH}$. Importantly, the carbon resonance at $181.6 \mathrm{ppm}$ shows a correlation with the $\mathrm{Zr}-\mathrm{OH}$ (Figure 3B), indicating the proximity of zirconol moieties to the carboxylate. Based on these observations, we depict the coordination modes in scheme 3 . The 189.3 carbonyl resonance corresponds to a chelating binding mode and the interpretation is straightforward. The most likely coordination for the bridging mode (181.6 ppm) is the situation where a carboxylate bridges a hydrogen and a zirconium atom (Scheme 3A). This satisfies the requirement for bridging coordination and takes into account the correlation to $\mathrm{Zr}-\mathrm{OH}$. However, on the basis of these data the presence of a carboxylate bridging two zirconium atoms, cannot be fully discarded (Scheme 3B). Nevertheless, in this case, a random position of the zirconol at the surface is expected and thus, some interactions (and the subsequent correlation in the HETCOR spectrum) should also be observed with the chelating carboxylates. The most exiting result is that the prevalent bridging coordination seems to confirm the surface chemistry binding motif $\mathrm{NC}(\mathrm{X})_{2}$ where two X-type moieties (proton and carboxylate) are interacting with two different surface sites (zirconium and oxygen). However, it should be noted that the present experiments also indicate that there is still a proton-carboxylate interaction and that the carboxylic acid is thus 
not completely dissociated. In this respect, one may argue that carboxylic acids can be regarded, not as a combination of $2 \mathrm{X}$-type ligands but rather one ligand with two X-type functionalities; $\mathrm{NC}\left(\mathrm{X}_{2}\right)$ instead of $\mathrm{NC}(\mathrm{X})_{2}$.

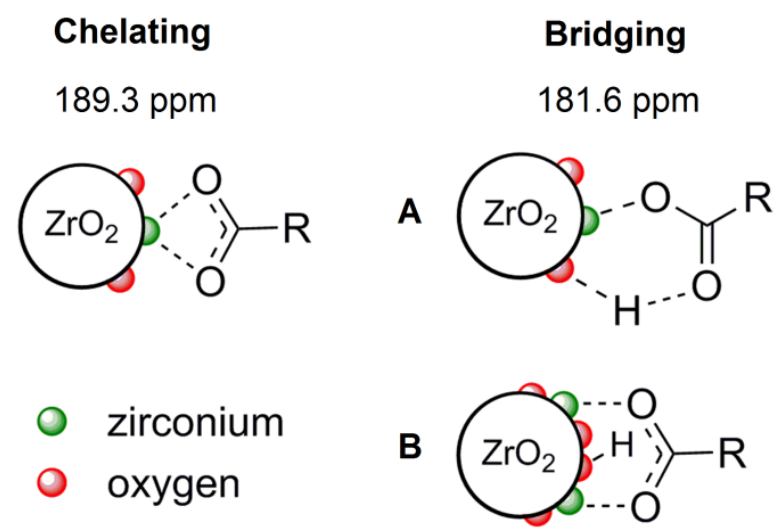

Scheme 3. The three possible binding modes on the surface of $\mathrm{ZrO}_{2} \mathrm{NCs}$.

Generalization to $\mathrm{HfO}_{2}$ NCs. The same experiments were conducted for $\mathrm{HfO}_{2} \mathrm{NCs}_{\text {. About }}$ the same frequency difference $\left(136 \mathrm{~cm}^{-1}\right)$ was found between the symmetric and the antisymmetric carbonyl stretch in the IR spectrum (Figure S3). Indeed, the coordination modes of dodecanoic acid towards the hafnia surface are identical as to the zirconia surface since the same carbonyl resonances are apparent from the CP MAS spectrum (Figure 4A). The $\alpha \mathrm{CH}_{2}$ resonance is again not detected in the INEPT experiment, confirming the orientation of dodecanoic acid to $\mathrm{HfO}_{2}$. The surface hydroxyl groups are clearly detected in the ${ }^{1} \mathrm{H}$ MAS spectrum (Figure 4B) and the correlation with the bridging mode is apparent from the HETCOR experiment (Figure 4C). Although HETCOR is powerful, the experiment is also time consuming. Recently, Delpech and coworkers have proposed the use of a Forth and Back $\mathrm{CP}$ experiment $(\mathrm{FBCP})$ where polarization is transferred from hydrogen to phosphorus atoms of a NC and back to hydrogen atoms. ${ }^{[29]}$ In this way, it was possible to selectively probe the hydrogen atoms on and close to the phosphorus atom at the $\mathrm{NC}$ surface. In our case of $\mathrm{HfO}_{2}$ NCs, the inorganic core does not contain any abundant NMR sensitive nucleus, hampering the technique. However, the carbonyl carbon is quite isolated in the ${ }^{13} \mathrm{C}$ NMR spectrum and therefore, it was possible with the use of selective pulses to edit hydrogen atoms close to carbonyl through selective polarization transfers. The surface hydroxyl groups are now clearly apparent in the ${ }^{1} \mathrm{H}$ FBCP NMR spectrum (Figure 4B). 


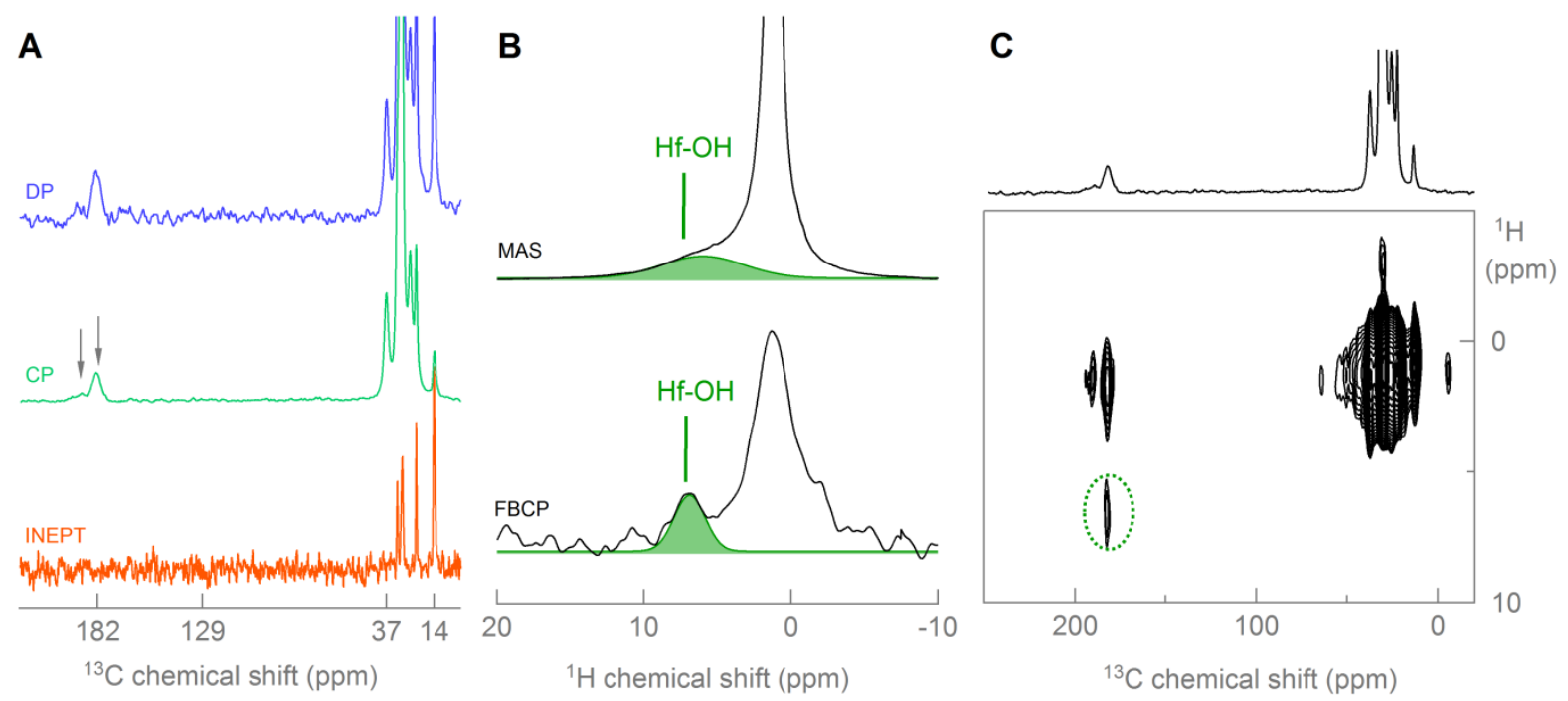

Figure 4. (A) ${ }^{13} \mathrm{C}$ DP,${ }^{1} \mathrm{H}_{-}{ }^{13} \mathrm{C} \mathrm{CP}$ and INEPT MAS spectra and (B) ${ }^{1} \mathrm{H}$ MAS and ${ }^{1} \mathrm{H}_{-}{ }^{13} \mathrm{C}-{ }^{1} \mathrm{H}$ FBCP MAS spectra and (C) HETCOR spectrum of $5 x$ purified $\mathrm{HfO}_{2} \mathrm{NCs}$ capped with dodecanoic acid.

Ligand exchange. It is known from literature that amine ligands are able to displace carboxylic acids on metal oxide surfaces when provided in a large excess. ${ }^{[11]}$ To investigate the influence of this ligand exchange to the coordination modes, we added 10 equivalents of oleylamine to the $5 \mathrm{x}$ purified $\mathrm{ZrO}_{2}$ and $\mathrm{HfO}_{2} \mathrm{NCs}$, dispersed in chloroform. This causes the displacement of $13 \%$ of the original carboxylate capping layer (Figure S4). We subsequently purified the dispersion three times with acetone and investigated the resulting NC powder and the first supernatant. Although the IR spectra before and after amine treatment look identical (Figures S5 and S6), the ssNMR spectra are more revealing. It appears that the chelating mode has vanished in the case of $\mathrm{ZrO}_{2} \mathrm{NCs}$ and decreased considerably in intensity in the case of $\mathrm{HfO}_{2} \mathrm{NCs}$ (see Figure 5).

A

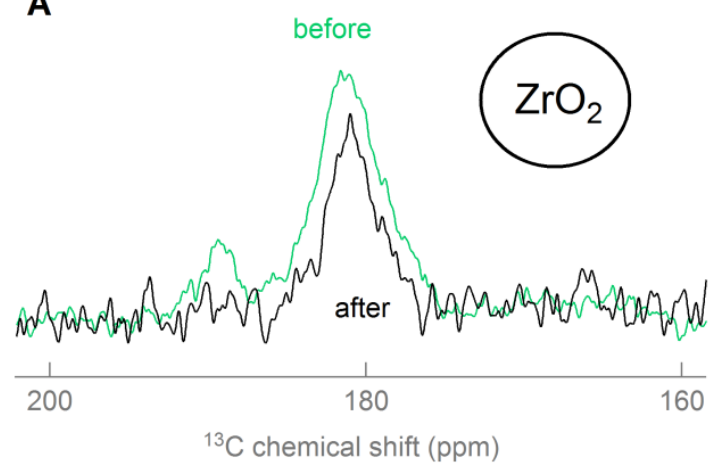

B

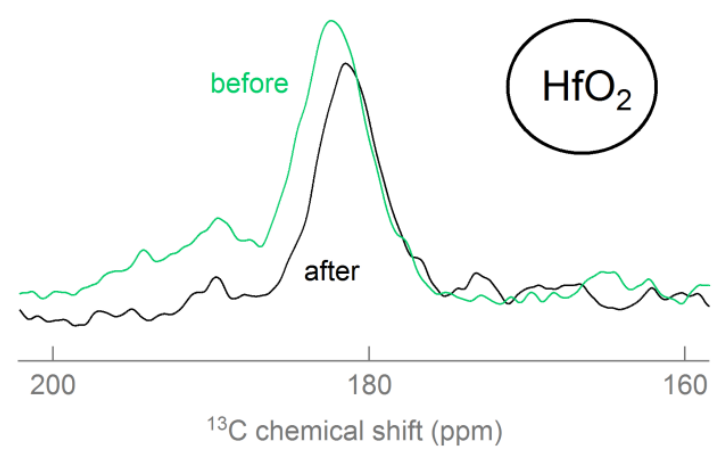

Figure 5. ${ }^{13} \mathrm{C} \mathrm{CP}$ MAS spectra of $\mathrm{ZrO}_{2}$ and $\mathrm{HfO}_{2} \mathrm{NCs}$ before and after addition of oleylamine and purification.

These results clearly show a higher reactivity of the carboxylate ligand in chelating mode than that one in bridging mode towards the ligand exchange by oleylamine. This selectivity in the 
reaction can be explained by the higher electrophilicity of the carboxylate moiety in the former mode compared to the latter. The different electrophilicity of these carboxylates is also supported by the difference in their chemical shift of the ${ }^{13} \mathrm{C}$ carboxylate nuclei (189.3 vs. $181.6 \mathrm{ppm}$ for chelating and bridging mode, respectively vide supra). This is attributed to electronic effects of the bound metal. ${ }^{[27]}$

Nanocatalysis. Analysis of the supernatant revealed the formation of an imine product (see Figure 6). This is derived from the condensation reaction between oleylamine and acetone; the solvent used for the precipitation of both $\mathrm{ZrO}_{2}$ and $\mathrm{HfO}_{2} \mathrm{NCs}$. The characterization of the imine is available in the supporting info (Figure S7). A higher imine content was found in the supernatant of the $\mathrm{HfO}_{2} \mathrm{NCs}$ as determined by solution ${ }^{1} \mathrm{H}$ NMR spectroscopy (95\% vs. 75\% for $\mathrm{HfO}_{2}$ and $\mathrm{ZrO}_{2}$ respectively). The conversion yield was calculated based on oleylamine. In order to confirm the nature of the reaction (NC-catalyzed or stoichiometric), a control experiment between oleylamine and acetone was performed, in the presence or absence of dodecanoic acid. Under the same conditions as the nano-catalysis (40 $\mathrm{min}$ at room temperature, [oleylamine] $=0.076 \mathrm{M}$ and [acetone] $=10 \mathrm{M}$ ), the reaction only proceeded 13 or $14 \%$, with or without dodecanoic acid respectively ([dodecanoic acid]=0.0076 M), on the basis of ${ }^{1} \mathrm{H}$ NMR spectroscopy. This result confirms that $\mathrm{ZrO}_{2}$ and $\mathrm{HfO}_{2} \mathrm{NCs}$ are acting as nano-catalysts in the condensation reaction, as it is well known that the synthesis of imines from carbonyl compounds can be acid-catalyzed. ${ }^{[30]}$ The control experiment with dodecanoic acid suggests that the reaction might be more likely catalyzed by the Lewis acid (hafnium or zirconium) surface sites rather than by the Brønsted acidity of adsorbed carboxylic acids, although the influence of hydrogen bonding cannot be excluded.

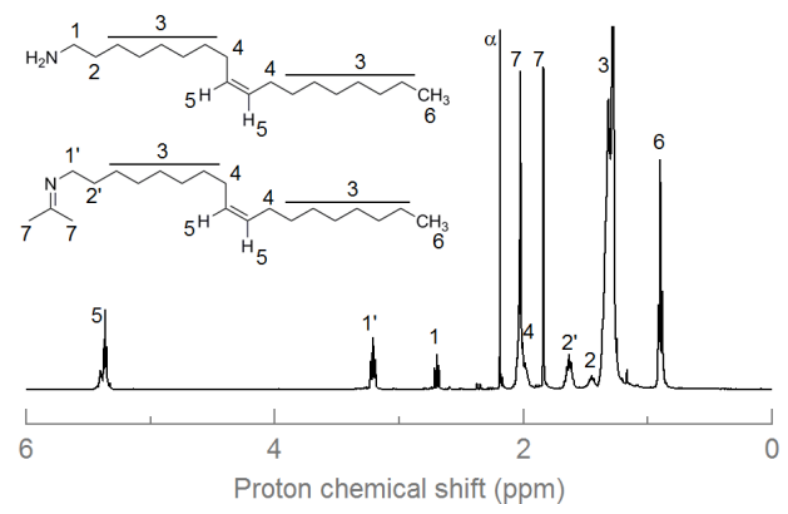

Figure 6. Solution ${ }^{1} \mathrm{H}$ NMR spectrum of the supernatant after purification of oleylamine treated $\mathrm{ZrO}_{2} \mathrm{NCs}$ with acetone, showing imine formation between acetone and oleylamine.

Imines are important intermediates in the synthesis of various biological, agricultural, and pharmaceutical compounds since they can undergo versatile transformations (reductions, 
additions, condensations, and multicomponent reactions. ${ }^{[30-31]}$ The high conversion percentage at room temperature, especially for $\mathrm{HfO}_{2}$, shows the potential of these metal oxides as nanocatalysts for this reaction. Although it should be noted that imines can be produced from alcohols and amines by using $\mathrm{Pd}$ or $\mathrm{Au}$ on a zirconia support, ${ }^{[32]}$ this is the first example (to the best of our knowledge) in which $\mathrm{ZrO}_{2}$ or $\mathrm{HfO}_{2}$ serve as the sole nano-catalyst for imine formation. Together with the recently discovered catalytic activity of $\mathrm{HfO}_{2}$ in the esterification and transesterification reaction, ${ }^{[2 \mathrm{a}]}$ these results put zirconia and hafnia squarely in the focus of acid-base catalysis and further optimization is required to bring these materials to their full potential.

\section{Conclusion}

Solid state NMR analysis was able to reveal unprecedented details on ligand shell configurations. We contributed to the discussion on secondary "entangled" ligands and show that those ligands are interacting with the surface through hydrogen bonding and electrostatic interaction. We also confirm the $\mathrm{NC}(\mathrm{X})_{2}$ binding motif of carboxylic acids on $\mathrm{ZrO}_{2}$ and $\mathrm{HfO}_{2}$ NC surfaces. Through the application of HETCOR and FBCP, the surface protons were identified and correlated to the carbonyl carbon of a carboxylate in a bridging coordination mode. Also a minor chelating mode was identified. During ligand exchange with oleylamine, the chelating mode appeared to be more reactive and was readily displaced. We also found that the zirconia and especially the hafnia NCs catalyzed the imine formation of acetone with oleylamine. These results confirm the importance of zirconia and hafnia for catalysis and widen the scope of their catalytic applications.

\section{Experimental section}

Nanocrystal synthesis. $\mathrm{HfO}_{2}$ and $\mathrm{ZrO}_{2} \mathrm{NCs}$ were synthesized via an established microwaveassisted solvothermal process. ${ }^{[10,21 \mathrm{~b}]}$ The precursor preparation was executed in a nitrogen filled glovebox. Very briefly, $0.5 \mathrm{~mL}$ of dibenzyl ether was added to $0.56 \mathrm{mmol}(0.13 \mathrm{~g})$ of $\mathrm{ZrCl}_{4}$, after which $4 \mathrm{~mL}$ of benzyl alcohol is added. After microwave heating at $210{ }^{\circ} \mathrm{C}$ for 3.5 hours, diethyl ether is added, causing phase separation. The particles are precipitated with ethanol and diethyl ether and washed once with diethyl ether. Finally the particles are 
redispersed in chloroform by the addition of $50 \mu \mathrm{L}$ oleic acid and $50 \mu \mathrm{L}$ oleylamine and ultrasonic treatment. The particles are purified five times with acetone. A more detailed description of synthesis procedure and work-up can be found elsewhere. ${ }^{[10]}$

Solution NMR Characterization. ${ }^{1} \mathrm{H}-,{ }^{13} \mathrm{C}-$, COSY, HSQC, and ${ }^{1} \mathrm{H}_{-}{ }^{13} \mathrm{C}$ HMBC-NMR spectra in solution were recorded on a Bruker Avance 500 NMR spectrometer. Chemical shifts $\left(\delta\right.$, parts per million) are quoted relative to $\mathrm{SiMe}_{4}\left({ }^{1} \mathrm{H},{ }^{13} \mathrm{C}\right)$. They were measured by internal referencing to the ${ }^{13} \mathrm{C}$ or residual ${ }^{1} \mathrm{H}$ resonances of the deuterated solvent $\left(\mathrm{CDCl}_{3}{ }^{1} \mathrm{H} \delta\right.$ 7.26; ${ }^{13} \mathrm{C} \delta 77.2$; respectively). Coupling constants $(J)$ are given in Hertz.

Solid State NMR Characterization. Solid-state NMR experiments were recorded on a Bruker Avance III spectrometers operating at magnetic fields of 9.4 T. Samples were packed into $3.2 \mathrm{~mm}$ zirconia rotors. The rotors were spun at $16 \mathrm{kHz}$ at room temperature. For ${ }^{1} \mathrm{H}$ MAS and ${ }^{13} \mathrm{C}$ DP MAS experiments, small flip angle $\left(\sim 30^{\circ}\right)$ were used with recycle delays of $5 \mathrm{~s}$ and $10 \mathrm{~s}$ respectively. ${ }^{13} \mathrm{C} \mathrm{CP}$ MAS were recorded with a recycle delay of $2 \mathrm{~s}$ and contact times between $2 \mathrm{~ms}$ and $5 \mathrm{~ms} .{ }^{13} \mathrm{C}$ INEPT were recorded with a recycle delay of $3 \mathrm{~s}$. The ${ }^{13} \mathrm{C}$ HETCOR were acquired with a contact time of $3 \mathrm{~ms}$ and a recycle delay of $1.5 \mathrm{~s}$. The Forth and Back $\mathrm{CP}$ (FBCP) experiment were conducted with a recycle delay of $1.5 \mathrm{~s}$, a first contact time (forth, ${ }^{1} \mathrm{H}->{ }^{13} \mathrm{C}$ ) and a second $\mathrm{CP}$ contact (back, ${ }^{13} \mathrm{C}->{ }^{1} \mathrm{H}$ ) of $0.5 \mathrm{~ms}$. Residual ${ }^{1} \mathrm{H}$ signals were eliminated with a saturation protocol that uses a loop of $\pi / 2$ pulses with a timedecreasing interval (from $100 \mathrm{~ms}$ to $10 \mathrm{~ms}$ by steps of $10 \mathrm{~ms}$ ). Carbonyl resonance were selected by replacing in the FBCP experiment, the second 90 degree hard pulse of ${ }^{13} \mathrm{C}$ by a 90 selective Gaussian shape pulse of $300 \mu$ s length. Chemical shifts were referenced to liquid TMS.

Other Characterization. FT-IR spectra were recorded on a Perkin-Elmer Spectrum 100 FTIR spectrometer (solid state samples). For X-ray diffraction (XRD) characterization a Thermo Scientific ARL X'tra X-ray diffractometer was used with the $\mathrm{CuK} \alpha$ line as the primary source. Transmission electron microscopy (TEM) images were taken on a JEOL JEM-2200FS TEM with Cs corrector.

\section{Acknowledgements}

This work was financially supported by the Research Foundation Flanders (FWO) and by the Agence Nationale pour la Recherche (Project ANR-13-IS10-0004-01). EAB is grateful to Marie Curie Actions and Campus France for a PRESTIGE post-doc fellowship (FP7/20072013) under REA grant agreement $n^{\circ}$ PCOFUND-GA-2013-609102. 


\section{Author contributions}

JDR synthesized the NCs and wrote the manuscript. YC carried out the NMR measurements. EAB carried out the IR measurements and wrote the manuscript. KDK performed TEM measurements. FD, CN supervised the research in Toulouse and FD wrote the manuscript. IVD and $\mathrm{ZH}$ supervised the research in Ghent. All authors analyzed the results and commented on the manuscript.

\section{Competing financial interests}

The authors declare no competing financial interests.

\section{References}

[1] a) Y. Yin, A. P. Alivisatos, Nature 2005, 437, 664-670; b) J. Lee, S. Zhang, S. H. Sun, Chem. Mat. 2013, 25, 1293-1304; c) J. Park, J. Joo, S. G. Kwon, Y. Jang, T. Hyeon, Angew. Chem.-Int. Edit. 2007, 46, 4630-4660; d) N. R. Jana, Y. F. Chen, X. G. Peng, Chem. Mat. 2004, 16, 3931-3935; e) D. Ito, S. Yokoyama, T. Zaikova, K. Masuko, J. E. Hutchison, ACS Nano 2014, 8, 64-75.

[2] a) J. De Roo, I. Van Driessche, J. C. Martins, Z. Hens, Nat. Mater. 2016, 15, 517-521; b) Z. Q. Niu, Y. D. Li, Chem. Mat. 2014, 26, 72-83; c) Z. Wu, D. E. Jiang, A. K. Mann, D. R. Mullins, Z. A. Qiao, L. F. Allard, C. Zeng, R. Jin, S. H. Overbury, J. Am. Chem. Soc. 2014, 136, 6111-6122.

[3] M. Ibáñez, R. J. Korkosz, Z. Luo, P. Riba, D. Cadavid, S. Ortega, A. Cabot, M. G. Kanatzidis, J. Am. Chem. Soc. 2015, 137, 4046-4049.

[4] a) M. Ibanez, Z. Luo, A. Genc, L. Piveteau, S. Ortega, D. Cadavid, O. Dobrozhan, Y. Liu, M. Nachtegaal, M. Zebarjadi, J. Arbiol, M. V. Kovalenko, A. Cabot, Nat. Commun. 2016, 7, 10766; b) P. Cayado, K. De Keukeleere, A. Garzón, L. PerezMirabet, A. Meledin, J. De Roo, F. Vallés, B. Mundet, H. Rijckaert, G. Pollefeyt, M. Coll, S. Ricart, A. Palau, J. Gázquez, J. Ros, G. Van Tendeloo, I. Van Driessche, T. Puig, X. Obradors, Supercond. Sci. Tech. 2015, 28, 124007; c) C. Liu, T. J. Hajagos, D. Kishpaugh, Y. Jin, W. Hu, Q. Chen, Q. Pei, Adv. Funct. Mater. 2015, 25, 46074616; d) G. Garnweitner, L. M. Goldenberg, O. V. Sakhno, M. Antonietti, M. Niederberger, J. Stumpe, Small 2007, 3, 1626-1632.

[5] a) G. H. Carey, I. J. Kramer, P. Kanjanaboos, G. Moreno-Bautista, O. Voznyy, L. Rollny, J. A. Tang, S. Hoogland, E. H. Sargent, ACS Nano 2014, 8, 11763-11769; b) D. K. Ko, A. Maurano, S. K. Suh, D. Kim, G. W. Hwang, J. C. Grossmann, V. Bulovic, M. G. Bawendi, ACS Nano 2016, 10, 3382-3388; c) K. Whitham, J. Yang, B. H. Savitzky, L. F. Kourkoutis, F. Wise, T. Hanrath, Nat. Mater. 2016, 15, 557-563; d) W. Walravens, J. De Roo, E. Drijvers, S. Ten Brinck, E. Solano, J. Dendooven, C. Detavernier, I. Infante, Z. Hens, ACS Nano 2016, 10, 6861-6870.

[6] a) X. Lan, O. Voznyy, F. P. Garcia de Arquer, M. Liu, J. Xu, A. H. Proppe, G. Walters, F. Fan, H. Tan, Z. Yang, S. Hoogland, E. H. Sargent, Nano Lett 2016, 16, 4630-4634; b) J. S. Lee, M. V. Kovalenko, J. Huang, D. S. Chung, D. V. Talapin, Nat. Nanotechnol. 2011, 6, 348-352; c) M. V. Kovalenko, M. Scheele, D. V. Talapin, Science 2009, 324, 1417-1420; d) N. C. Anderson, M. P. Hendricks, J. J. Choi, J. S. 
Owen, J. Am. Chem. Soc. 2013, 135, 18536-18548; e) M. Scheele, J. H. Engel, V. E. Ferry, D. Hanifi, Y. Liu, A. P. Alivisatos, ACS Nano 2013, 7, 6774-6781.

[7] A. Llordes, G. Garcia, J. Gazquez, D. J. Milliron, Nature 2013, 500, 323.

[8] a) Z. Hens, J. C. Martins, Chem. Mat. 2013, 25, 1211-1221; b) A. J. Morris-Cohen, M. Malicki, M. D. Peterson, J. W. J. Slavin, E. A. Weiss, Chem. Mat. 2013, 25, 11551165; c) L. E. Marbella, J. E. Millstone, Chem. Mat. 2015, 27, 2721-2739; d) C. Bonhomme, C. Gervais, D. Laurencin, Prog. Nucl. Magn. Reson. Spectrosc. 2014, 77, $1-48$.

[9] I. Moreels, B. Fritzinger, J. C. Martins, Z. Hens, J. Am. Chem. Soc. 2008, 130, 1508115086.

[10] J. De Roo, F. Van den Broeck, K. De Keukeleere, J. C. Martins, I. Van Driessche, Z. Hens, J. Am. Chem. Soc. 2014, 136, 9650-9657.

[11] J. De Roo, Y. Justo, K. De Keukeleere, F. Van den Broeck, J. C. Martins, I. Van Driessche, Z. Hens, Angew. Chem.-Int. Edit. 2015, 54, 6488-6491.

[12] a) C. N. Valdez, M. Braten, A. Soria, D. R. Gamelin, J. M. Mayer, J. Am. Chem. Soc. 2013, 135, 8492-8495; b) J. N. Schrauben, R. Hayoun, C. N. Valdez, M. Braten, L. Fridley, J. M. Mayer, Science 2012, 336, 1298-1301.

[13] J. De Roo, M. Ibáñez, P. Geiregat, G. Nedelcu, W. Walravens, J. Maes, J. C. Martins, I. Van Driessche, M. V. Kovalenko, Z. Hens, ACS Nano 2016, 10, 2071-2081.

[14] J. De Roo, K. De Keukeleere, Z. Hens, I. Van Driessche, Dalton Trans. 2016, 45, 13277-13283.

[15] J. De Roo, S. Coucke, H. Rijckaert, K. De Keukeleere, D. Sinnaeve, Z. Hens, J. C. Martins, I. Van Driessche, Langmuir 2016, 32, 1962-1970.

[16] a) N. C. Anderson, J. S. Owen, Chem. Mat. 2013, 25, 69-76; b) M. H. Mobarok, J. M. Buriak, Chem. Mat. 2014, 26, 4653-4661; c) A. Hassinen, I. Moreels, K. De Nolf, P. F. Smet, J. C. Martins, Z. Hens, J. Am. Chem. Soc. 2012, 134, 20705-20712; d) E. L. Rosen, R. Buonsanti, A. Llordes, A. M. Sawvel, D. J. Milliron, B. A. Helms, Angew. Chem.-Int. Edit. 2012, 51, 684-689; e) T. Gutmann, E. Bonnefille, H. Breitzke, P.-J. Debouttiere, K. Philippot, R. Poteau, G. Buntkowsky, B. Chaudret, Phys. Chem. Chem. Phys. 2013, 15, 17383-17394; f) T. Pery, K. Pelzer, G. Buntkowsky, K. Philippot, H.-H. Limbach, B. Chaudret, ChemPhysChem 2005, 6, 605-607.

[17] a) I. Villa, A. Vedda, M. Fasoli, R. Lorenzi, N. Kränzlin, F. Rechberger, G. Ilari, D. Primc, B. Hattendorf, F. J. Heiligtag, M. Niederberger, A. Lauria, Chem. Mat. 2016, 28, 3245-3253; b) A. Lauria, I. Villa, M. Fasoli, M. Niederberger, A. Vedda, ACS Nano 2013, 7, 7041-7052.

[18] L. Maggiorella, G. Barouch, C. Devaux, A. Pottier, E. Deutsch, J. Bourhis, E. Borghi, L. Levy, Future Oncol. 2012, 8, 1167-1181.

[19] a) T. A. Cheema, G. Garnweitner, Crystengcomm 2014, 16, 3366-3375; b) C. Liu, T. J. Hajagos, D. Chen, Y. Chen, D. Kishpaugh, Q. Pei, ACS Appl. Mater. Interfaces 2016, 8, 4795-4802; c) J. Molina, A. L. Munoz, W. Calleja, P. Rosales, A. Torres, J. Mat. Sci. 2012, 47, 2248-2255.

[20] a) T. Finke, D. Lingenfelser, K. Bindler, U. Eisele, H. Bockhorn, G. Brunklaus, J. Am. Ceram. Soc. 2009, 92, 1823-1830; b) R. Ramos-González, L. A. García-Cerda, M. A. Quevedo-López, Appl. Surf. Sci. 2012, 258, 6034-6039.

[21] a) J. De Roo, K. De Keukeleere, J. Feys, P. Lommens, Z. Hens, I. Van Driessche, J. Nanopart. Res. 2013, 15, 1778; b) K. De Keukeleere, J. De Roo, P. Lommens, J. C. Martins, P. Van der Voort, I. Van Driessche, Inorg. Chem. 2015, 54, 3469-3476.

[22] a) C. Grote, T. A. Cheema, G. Garnweitner, Langmuir 2012, 28, 14395-14404; b) C. Grote, K. J. Chiad, D. Vollmer, G. Garnweitner, Chem. Commun. 2012, 48, 14641466. 
[23] B. Fritzinger, R. K. Capek, K. Lambert, J. C. Martins, Z. Hens, J. Am. Chem. Soc. 2010, 132, 10195-10201.

[24] a) A. Cros-Gagneux, F. Delpech, C. Nayral, A. Cornejo, Y. Coppel, B. Chaudret, J. Am. Chem. Soc. 2010, 132, 18147-18157; b) W. S. Ojo, S. Xu, F. Delpech, C. Nayral, B. Chaudret, Angew. Chem.-Int. Edit. 2012, 51, 738-741; c) N. El Hawi, C. Nayral, F. Delpech, Y. Coppel, A. Cornejo, A. Castel, B. Chaudret, Langmuir 2009, 25, 75407546; d) A. Cornejo, G. Fuks, V. Martinez-Merino, I. Sarobe, M. J. Gil, K. Philippot, B. Chaudret, F. Delpech, C. Nayral, New J. Chem. 2014, 38, 6103-6113; e) S. Soule, J. Allouche, J. C. Dupin, C. Courreges, F. Plantier, W. S. Ojo, Y. Coppel, C. Nayral, F. Delpech, H. Martinez, Phys. Chem. Chem. Phys. 2015, 17, 28719-28728.

[25] A. Nowacka, P. C. Mohr, J. Norrman, R. W. Martin, D. Topgaard, Langmuir 2010, 26, 16848-16856.

[26] K. Nakamoto, Infrared and Raman Spectra of Inorganic and Coordination Compounds, Wiley-Interscience, New York, 1978.

[27] B.-H. Ye, X.-Y. Li, I. D. Williams, X.-M. Chen, Inorg. Chem. 2002, 41, 6426-6431.

[28] a) F. Faccini, H. Fric, U. Schubert, E. Wendel, O. Tsetsgee, K. Muller, H. Bertagnolli, A. Venzo, S. Gross, J. Mater. Chem. 2007, 17, 3297-3307; b) M. Puchberger, F. R. Kogler, M. Jupa, S. Gross, H. Fric, G. Kickelbick, U. Schubert, Eur. J. Inorg. Chem. 2006, 3283-3293; c) F. R. Kogler, M. Jupa, M. Puchberger, U. Schubert, J. Mater. Chem. 2004, 14, 3133-3138.

[29] E. A. Baquero, W.-S. Ojo, Y. Coppel, B. Chaudret, B. Urbaszek, C. Nayral, F. Delpech, Phys. Chem. Chem. Phys. 2016, 18, 17330-17334.

[30] R. D. Patil, S. Adimurthy, Asian J. Org. Chem. 2013, 2, 726-744.

[31] a) J. Gawronski, N. Wascinska, J. Gajewy, Chem. Rev. 2008, 108, 5227-5252; b) R. W. Layer, Chem. Rev. 1963, 63, 489-510.

[32] a) W. Cui, B. Zhaorigetu, M. Jia, W. Ao, H. Zhu, RSC Adv. 2014, 4, 2601-2604; b) J. W. Zheng, J. Y. Li, H. L. Wei, J. Yu, H. Q. Su, X. J. Wang, Mat. Sci Semicon. Proc. 2015, 32, 131-136; c) W. J. Cui, H. Y. Zhu, M. L. Jia, W. L. Ao, Y. L. Zhang, B. Zhaorigetu, React. Kinet. Mech. Cat 2013, 109, 551-562. 


\section{TOC entry}

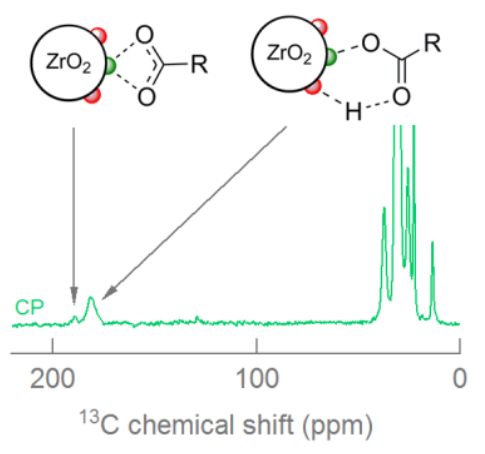

\section{TOC text}

Solid state NMR showed that entangled carboxylic acid ligands are interacting with the nanocrystal surface through hydrogen bonding and electrostatic interaction. Tightly bound carboxylic acids are mostly adsorbed on the NC surface with both proton and carboxylate. Also a minor chelating mode was identified. 